\title{
Perfusión cerebral por tomografía computada: Utilidad más allá del infarto cerebral agudo
}

Luis Canales F..", Beatriz Pérez O. $^{2}$, José de Grazia K. ${ }^{3}$, Lorena López G. ${ }^{4}$, Gonzalo Miranda G. ${ }^{3}$, Patricia Orellana $P_{.}{ }^{3}$, Alejandra Torres G. $^{3}$, Álvaro Salas $Z .^{3}$, René Núñez $F^{3}$, Marcelo López R. $^{3}$

1. Médico Neurorradiólogo, Unidad de Imagenología, Hospital Clínico Herminda Martín, Chillán, Chile.

2. Residente de Radiología, Departamento de Radiología, Universidad de Chile. Santiago, Chile.

3. Médico Neurorradiólogo, Centro de Imagenología del Hospital Clínico Universidad de Chile. Santiago, Chile.

4. Tecnóloga Médica, Centro de Imagenología del Hospital Clínico Universidad de Chile. Santiago, Chile.

\section{Cerebral perfusion CT: Utility beyond acute cerebral infarct}

\section{Resumen}

La perfusión cerebral por tomografía computada (PTC) es una técnica radiológica que permite una rápida evaluación cualitativa y cuantitativa de la perfusión cerebral mediante la generación de mapas de colores que representan el comportamiento de la microcirculación cerebral. La principal indicación es el estudio del infarto cerebral agudo para determinar la penumbra isquémica susceptible de terapia de reperfusión. Sin embargo, hay evidencia de su utilidad en otras situaciones, tales como: estados de hipo e hiperperfusión, determinación del grado de los tumores gliales, traumatismos encefálicos y otros. Las ventajas de la PTC son su disponibilidad, rapidez y la posibilidad de realizarla en conjunto con el estudio angiográfico. Sus principales desventajas derivan del uso de radiación ionizante y medio de contraste yodado. Presentamos diferentes entidades clínicas que se han estudiado en nuestra institución con PTC, donde esta técnica ha proporcionado información relevante para el diagnóstico y tratamiento. Con base en los parámetros de perfusión cerebral, proponemos una tabla para la interpretación de los diferentes mapas y los valores cuantitativos, con puntos de corte que definen los estados de normalidad, flujo lento, hipoperfusión, isquemia, infarto e hiperperfusión.

Palabras clave: Circulación cerebrovascular; Imágenes de perfusión; Perfusión cerebral; Tomografía computada.

\begin{abstract}
Computed tomography perfusion (CTP) is a technique that allows rapid qualitative and quantitative evaluation of cerebral perfusion by generating color maps that represent the behavior of the cerebral microcirculation. Its main indication has been the study of acute cerebral infarction in order to determine ischemic penumbra susceptible to reperfusion therapy. However, there is evidence of its usefulness in other situations such as: hypo - hyperperfusion states, tumor grading in glial neoplasms, head trauma and others. The advantages of CTP are its availability, speed and the possibility of performing it in conjunction with angiographic studies. However, it has disadvantages derived from the use of ionizing radiation and iodinated contrast agent. We present different clinical entities that have been studied in our institution with CTP, where the technique has provided relevant information for diagnosis and treatment. We propose a summary table for the interpretation of the different maps and the quantitative values, with cut points that define normality, slow flow, hypoperfusion, ischemia, core infarct and hyperperfusion.

Keywords: Cerebral perfusion CT; Cerebrovascular circulation; Computed tomography; Perfusion imaging.
\end{abstract}

Canales L., et al. Perfusión cerebral por tomografía computada: Utilidad más alla del infarto cerebral agudo. Rev Chil Radiol 2021; 27(1): 27-39.

*Correspondencia: Luis Canales F. / luiscanalesf@gmail.com

Trabajo enviado el 11 de noviembre de 2019. Aceptado para publicación el 15 de agosto de 2020. 


\section{Introducción}

La perfusión cerebral por TC (PTC) es una técnica que permite una rápida evaluación cualitativa y cuantitativa de la perfusión cerebral, mediante la generación de mapas de colores que representan el comportamiento de la microcirculación cerebral, junto con obtener valores numéricos de los principales indicadores hemodinámicos cerebrales ${ }^{1,2}$. Su principal indicación ha sido el estudio del infarto cerebral agudo, con el objetivo de demostrar penumbra isquémica susceptible de terapia de reperfusión ${ }^{2}$, sin embargo, existe evidencia de su utilidad en otras situaciones clínicas como estados de hipo e hiperperfusión, determinación del grado en tumores gliales, vasculopatías, traumatismo encefálico, entre otros ${ }^{1,3}$.

Las ventajas de esta técnica son su disponibilidad, su rapidez y la posibilidad de efectuarla en forma conjunta con estudios angiográficos ${ }^{4,6}$. Además, otro punto a su favor es que se trata de una técnica de estudio de perfusión cerebral que permite obtener resultados cuantitativos (valores numéricos), lo cual la diferencia por ejemplo de la mayoría de las técnicas de perfusión cerebral por RM, donde solo se obtienen resultados cualitativos (curvas y mapas de colores, sin valores numéricos) ${ }^{4}$. La PTC presenta sin embargo algunas desventajas, derivadas principalmente del uso de radiación ionizante y medio de contraste yodado ${ }^{2,4}$.

En el presente artículo se resumen las principales características de la PTC, se enfatizan algunos conceptos hemodinámicos y se propone una tabla para la interpretación de los diferentes mapas y los valores cuantitativos (con puntos de corte que definen los estados de normalidad, flujo lento, hipoperfusión, isquemia, infarto e hiperperfusión). Finalmente, se repasan las indicaciones actuales de la técnica y se ejemplifica cada una de ellas con casos ilustrativos de nuestra serie, interpretados de acuerdo a la pauta propuesta.

\section{Aspectos técnicos}

\section{Adquisición y reducción de dosis}

En nuestra institución las exploraciones de PTC se efectúan utilizando un equipo Siemens, Somatom Definition $\mathrm{Edge}^{\circledR}$, de 128 canales de detección, con los parámetros técnicos que se resumen en la tabla 1. El procedimiento contempla la adquisición de una TC cerebral sin medio de contraste, seguida de la adquisición de perfusión, esta última con un rango de corte máximo de $10 \mathrm{~cm}$ en el eje caudo-craneal (lo cual condiciona que se deba focalizar el estudio a áreas o territorios vasculares de interés). Si se requiere estudio angiográfico complementario, éste se realiza posterior a la $\mathrm{PTC}^{4,7}$.

El ajuste de los parámetros de mAs y $\mathrm{kV}$ a valores de 180 y 70 respectivamente, nos ha permitido realizar estudios de PTC de buena calidad, con dosis de radiación aceptables. Ocupando estos parámetros obtenemos valores de DLP cercanos a 1.500 mGycm, lo que resulta comparable a la dosis de un Angio-TC de cuello y cerebro. La DLP total también se reduce al eliminar las fases adicionales (estudio sin medio de contraste y estudio angiográfico) en pacientes que ya tienen otras técnicas de imagen realizadas previamente ${ }^{8,9}$.

Tabla 1. Parámetros técnicos del estudio de perfusión cerebral por TC.

\begin{tabular}{|c|c|}
\hline Parámetro & Valor o descripción \\
\hline Adquisición & Helicoidal \\
\hline Dirección & Caudo-craneal \\
\hline $\begin{array}{l}\text { Posicionamiento } \\
\text { (plano de corte) }\end{array}$ & $\begin{array}{l}\text { Paralelo a la base de } \\
\text { cráneo }\end{array}$ \\
\hline $\mathrm{kV}$ & 70 \\
\hline $\mathrm{mAs}$ & 180 \\
\hline Colimación & $128 \times 0,6 \mathrm{~mm}$ \\
\hline Tiempo de rotación & $0,28 \mathrm{seg}$ \\
\hline Rango de adquisición & $100 \mathrm{~mm}$ \\
\hline \multicolumn{2}{|l|}{ Medio de contraste } \\
\hline •Punción & $\begin{array}{l}\text { Antecubital, aguja } \\
\text { de } 18 \mathrm{G}\end{array}$ \\
\hline - Volumen & $40 \mathrm{ml}$ \\
\hline - Retardo & 4 seg \\
\hline • Flujo de inyección & $6 \mathrm{ml} / \mathrm{seg}$ \\
\hline $\begin{array}{l}\text { Filtro Kernel de reconstrucción } \\
\text { Reconstrucción }\end{array}$ & $\begin{array}{l}\mathrm{H} 20 \\
1 / 1 \mathrm{~mm}\end{array}$ \\
\hline
\end{tabular}

\section{Posprocesamiento}

La técnica se fundamenta en la monitorización del primer paso de un bolo de medio de contraste a través de la microcirculación cerebral, lo que determina un incremento transitorio en la atenuación de los rayos $\mathrm{X}$, directamente proporcional a la cantidad de medio de contraste presente en los vasos de la región estudiada", ${ }^{1,3}$. Esto genera curvas de "tiempo-atenuación", las que representan los cambios transitorios en la atenuación producidos en cada vóxel durante la circulación del bolo de medio de contraste a través de los sistemas arterial y venoso. Morfológicamente la curva arterial suele ser más precoz, baja y aguda en relación a la curva venosa. La circulación arterial y venosa deben ser previamente seleccionadas a través de un ROI (region of interest) arterial y un ROI venoso, utilizando habitualmente la arteria cerebral anterior o media para el input arterial, y la tórcula, 
seno sagital superior o transverso para el output venoso (Figura 1). La selección de estos ROI con la tecnología actual es habitualmente automática, sin embargo, la opción manual permite corregir defectos determinados de errores por volumen parcial. Las curvas de "tiempo-atenuación" permiten el cálculo de los parámetros de perfusión, basado en modelos matemáticos ${ }^{1,2,3,4}$. Dentro de los modelos más utilizados por los fabricantes se cuentan: el método de momentos, el método de la pendiente y el método de deconvolución (los fundamentos técnicos de cada uno de estos métodos sobrepasan el objetivo de este artículo). En nuestro centro utilizamos el método de deconvolución, que nos permite obtener el tiempo de tránsito medio (TTM) y el volumen sanguíneo cerebral (VSC). A partir del principio del volumen central se obtiene el flujo sanguíneo cerebral (FSC), considerando la ecuación FSC $=$ VSC $/$ TTM $^{4,5}$.

\section{Parámetros y mapas}

El postproceso de las curvas "tiempo-atenuación" permite la generación de mapas de colores que representan el comportamiento de la microcirculación cerebral. Estos mapas se muestran en una escala de colores relacionada con valores de corte establecidos para cada parámetro. De ese modo existe una representación simétrica de los hemisferios, con diferencias entre la sustancia blanca y sustancia gris, donde esta última presenta valores más altos de FSC y VSC, pero valores menores de TTM y tiempo de drenaje venoso (TTD), lo que se encuentra en directa relación con su mayor consumo energético ${ }^{2,4}$.

Los principales mapas son los de FSC, VSC y TTM. Existen mapas adicionales entre los que destacamos el TTD y el producto de extracción de flujo (PEF). El tiempo al máximo (TMax) es un parámetro que ha ganado terreno, dada su incorporación en los estudios de ventana terapéutica extendida en accidente cerebrovascular isquémico como el DEFUSE y DAWN ${ }^{4,6}$.

\section{Definiciones}

Flujo sanguíneo cerebral (FSC): Cantidad de sangre que pasa por $100 \mathrm{~g}$ de tejido cerebral en un minuto (mL/100g/min). Rango normal 55-100 $\mathrm{mL} / 100 \mathrm{~g} / \mathrm{min}^{2}$.

Volumen sanguíneo cerebral (VSC): Cantidad de sangre por $100 \mathrm{~g}$ de tejido (mL/100g). Rango normal $2-3 \mathrm{~mL} / 100 \mathrm{~g}^{2}$.

Tiempo de tránsito medio (TTM): Tiempo promedio que tarda la sangre en circular a través de

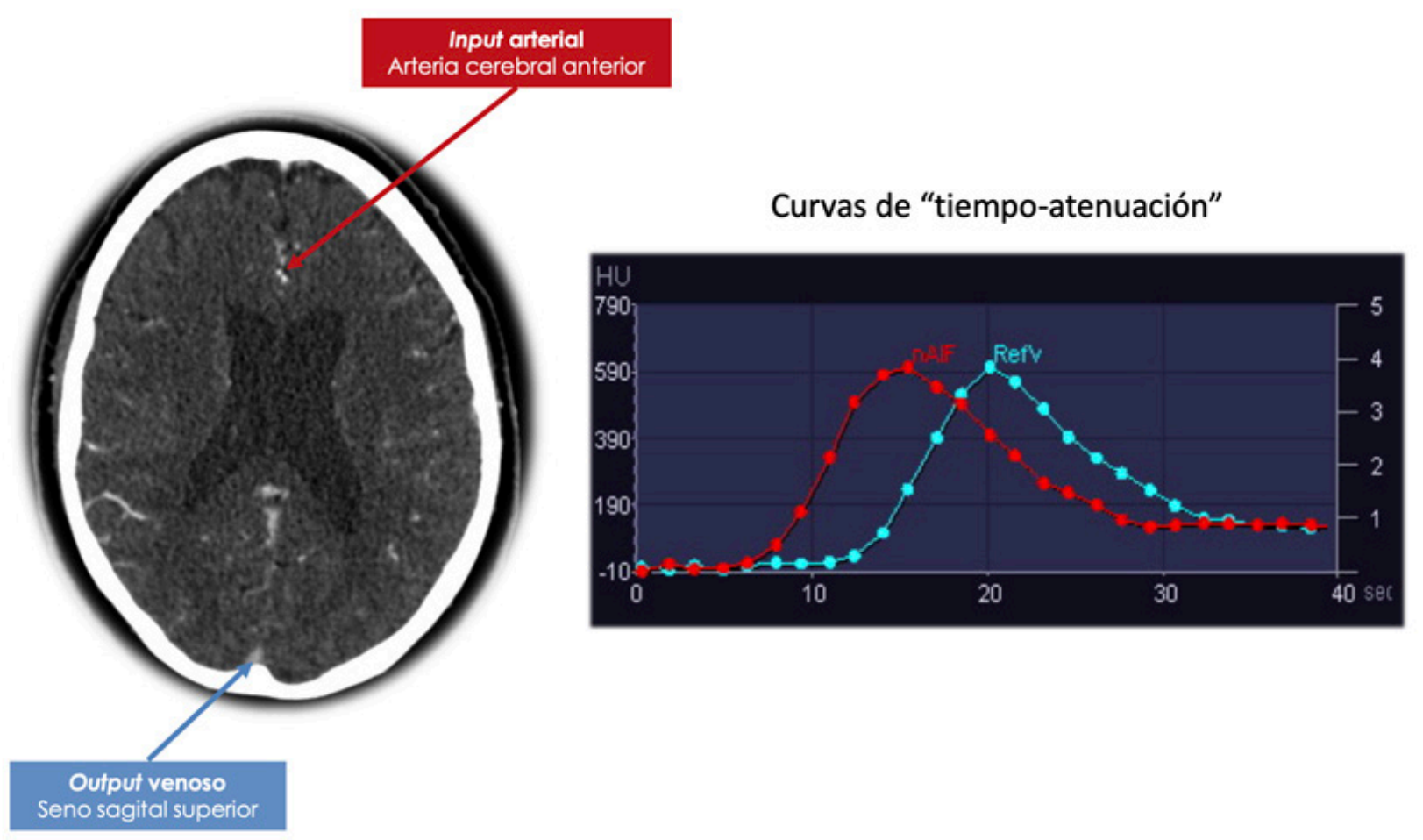

Figura 1: Selección de la circulación arterial y venosa: ROI (región of interest) para seleccionar el input arterial en la arteria cerebral anterior (señalado en rojo) y ROI para seleccionar el output venoso en el seno sagital superior (señalado en azul). Curvas de "tiempo-atenuación": Representan los cambios transitorios en la atenuación (densidad) producidos en cada vóxel durante la circulación del bolo de medio de contraste a través de los sistemas arterial y venoso (curvas roja y azul respectivamente). 
la vasculatura cerebral, desde la entrada arterial hasta la salida venosa. Rango normal $<6 \mathrm{seg}^{2}$.

Tiempo de drenaje venoso (TTD): Indicador del tiempo de lavado del medio de contraste.

Producto de extracción de flujo (PEF): Indicador de disrupción de la barrera hematoencefálica, con filtración de plasma al espacio intersticial.

En la figura 2 se muestran los conceptos de FSC, VSC y TTM, con una representación esquemática de cada uno, su definición y su rango de valor normal.

La figura 3 muestra los principales mapas de colores de un estudio de PTC (FSC, VSC y TTM), también los dos principales mapas adicionales (TTD y PEF) útiles para la evaluación clínica de la perfusión cerebral.

De acuerdo al valor del FSC es posible definir estados como hipoperfusión, oligoemia, isquemia, penumbra y core de infarto (Figura 4).

Para sintetizar el marco teórico y los puntos de corte de los parámetros VSC, FSC y TTM, proponemos en este artículo una tabla que establece cinco estados de perfusión cerebral: normalidad, flujo lento, hipoperfusión, isquemia, infarto e hiperperfusión (Tabla 2). Cada uno de estos estados pueden estar presentes en distintos escenarios clínicos. Conocer estos estados y los puntos de corte que los definen, resulta útil para una aproximación sistematizada al análisis de los estudios de PTC.

\section{Fuentes de error}

La PTC tiene limitaciones determinadas por la tecnología disponible y factores relativos a las etapas de adquisición, postproceso e interpretación, las que deben ser consideradas para evitar diagnóstico incorrecto ${ }^{4}$. Mencionamos algunas fuentes de error importantes:

- Rango de adquisición: El rango de corte máximo depende del equipamiento disponible, que oscila desde los $2,8 \mathrm{~cm}$ en tomógrafos de 64 canales de detección, $10 \mathrm{~cm}$ para equipos de 128 canales de detección y hasta la totalidad del encéfalo en equipos de 256 y 320 canales de detección. Esto favorece la interpretación y disminuye los falsos negativos ${ }^{9,10}$.

- Selección del input arterial y el output venoso, posicionamiento del paciente: La adecuada selección del input arterial y el output venoso es crítica para obtener mapas de PTC válidos. Esta etapa es habitualmente automatizada, sin embargo, debe ser revisada por el operador y corregida en caso necesario ya que puede significar una importante fuente de error. Asimismo, el correcto centrado del paciente en el momento de la adquisición es crucial para obtener mapas simétricos que faciliten la interpretación comparativa ${ }^{11,12}$.

- Resolución espacial: Los mapas de PTC tienen una resolución espacial relativamente baja, los

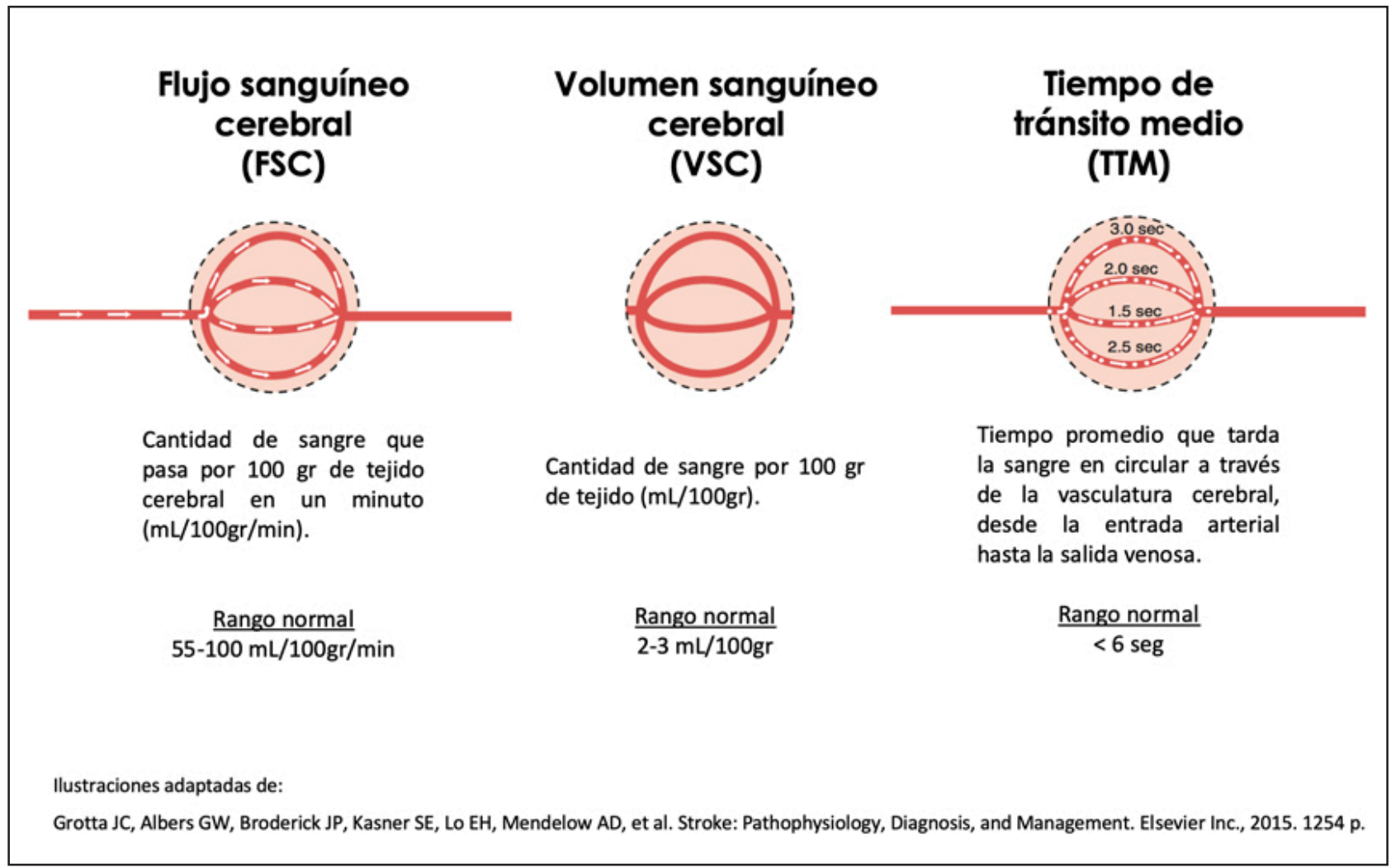

Figura 2: Representación esquemática, definición y rango de valor normal del flujo sanguíneo cerebral, volumen sanguíneo cerebral y tiempo de tránsito medio. 
infartos pequeños pueden ser omitidos incluso cuando se incluyen en el volumen interrogado; sin embargo, estos infartos pueden ser altamente sintomáticos cuando se ubican en áreas elocuentes. Las áreas de infarto crónico generalmente son obvias en la TC sin contraste; sin embargo, en la perfusión pueden ser confusas. La mayoría de los tejidos con infarto crónico muestran un grado bajo pero persistente de metabolismo y parámetros de perfusión disminuida, pero medible 4 .

- Retraso en el bolo de contraste: Dado que la adquisición del estudio se efectúa en un tiempo acotado, los factores que retrasan el arribo del bolo de contraste al encéfalo pueden conducir a errores de interpretación, especialmente para softwares automáticos (ej: Rapid®, Brainomix $\left.{ }^{\circledR}\right)$. Entre los factores se incluyen arritmias, insuficiencia cardíaca de bajo gasto, hipovolemia o estenosis carotídeas cervicales. El diagnóstico anticipado de estas condiciones y contar con estudios vasculares previos o contemporáneos, permiten corregir adecuadamente la interpretación ${ }^{13}$.

- Fenómeno del núcleo fantasma: Diversas investigaciones han demostrado una sobreestimación del núcleo del infarto cuando la PTC es realizada en las primeras 3 horas del inicio de los síntomas, especialmente cuando se considera el VSC, pero también para el FSC, lo que puede alterar la selección terapéutica de los pacientes. El ajuste de los umbrales de detección en el postproceso del examen, con el objetivo de hacerlos más exigentes (por ejemplo $<1,5 \mathrm{ml} / 100 \mathrm{~g}$ para el VSC), puede aumentar el valor predictivo positivo de los mapas obtenidos ${ }^{14}$.

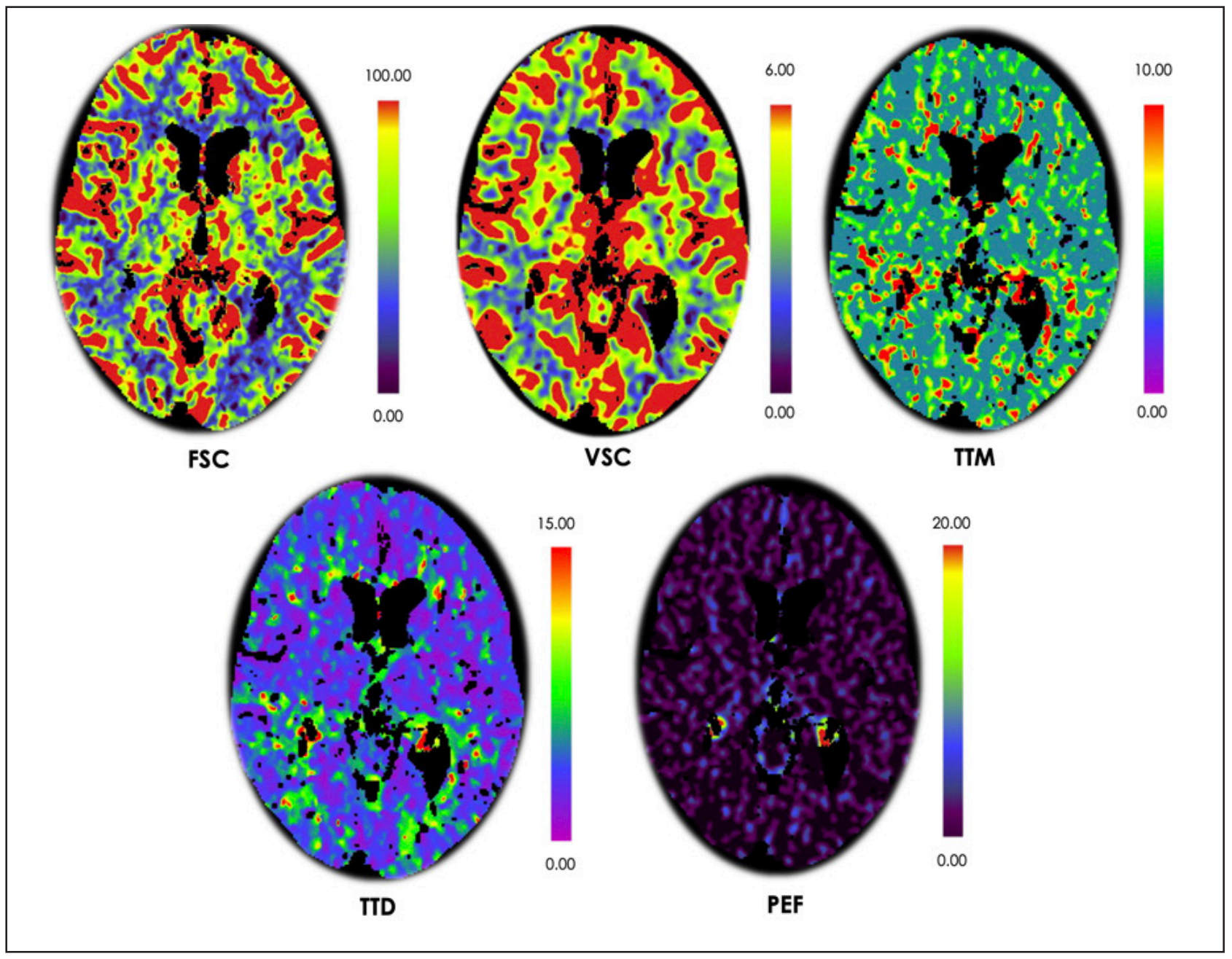

Figura 3: Principales mapas de perfusión cerebral: Flujo sanguíneo cerebral (FSC), volumen sanguíneo cerebral (VSC) y tiempo de tránsito medio (TTM). Se muestran además dos mapas adicionales: Tiempo de drenaje venoso (TTD) y producto de extracción de flujo (PEF). En este paciente sano, los mapas son simétricos, con valores en rango normal para cada parámetro. Nótese los mayores valores de FSC y VSC en la sustancia gris respecto a la sustancia blanca, también los tiempos (TTM y TTD) algo mayores en la sustancia blanca que en la sustancia gris. Por último, obsérvese el PEF, con valores altos solo en los plexos coroideos (estructuras que carecen de barrera hematoencefálica). 


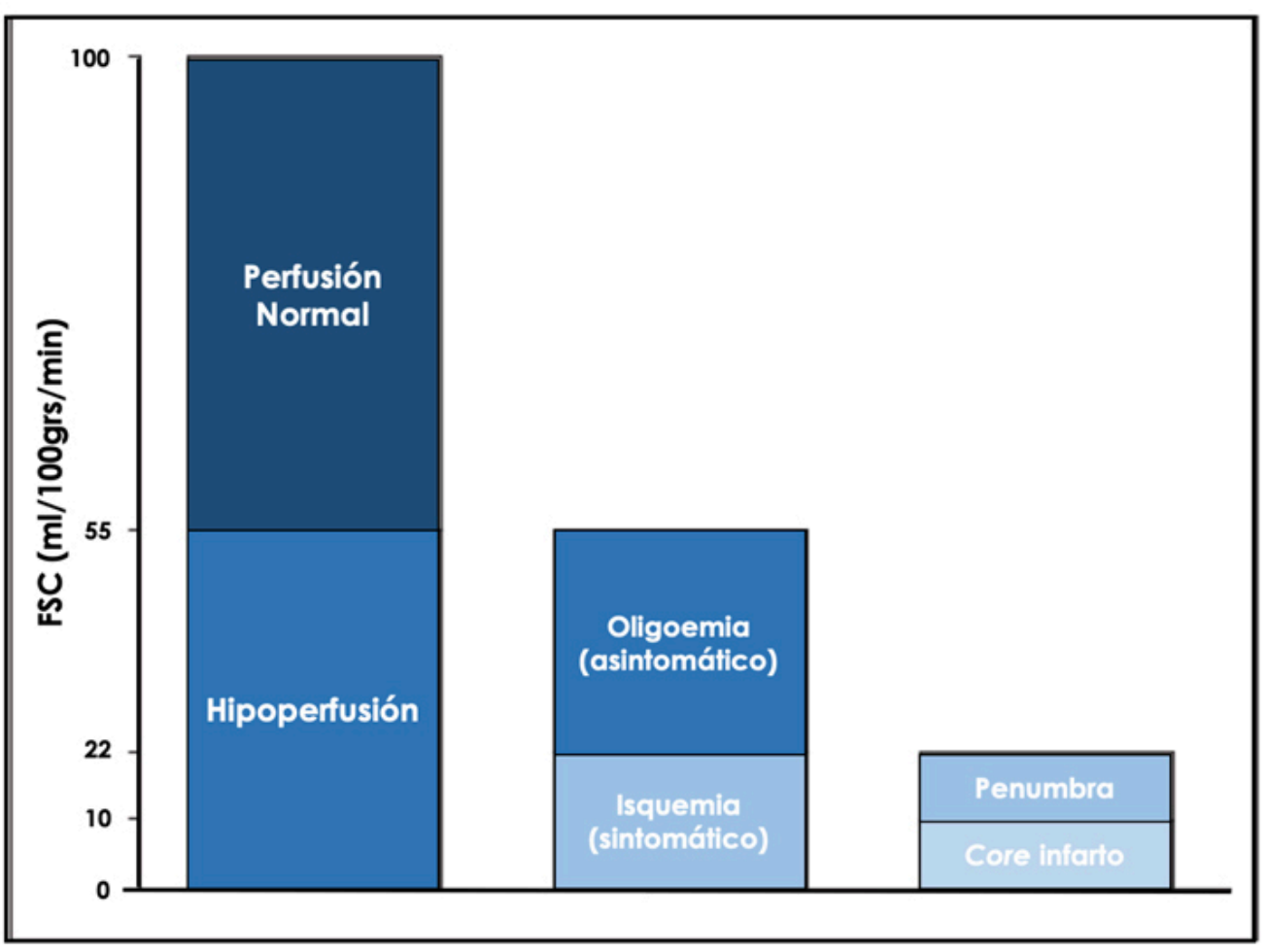

Gráfico adaptado de:

Yuh W, Alexander MD, Ueda T, Maeda M, Taoka T, Yamada K, et al. Revisiting Current Golden Rules in Managing Acute Ischemic Stroke: Evaluation of New Strategies to Further Improve Treatment Selection and Outcome. AJR Am J Roentgenol. 2017; 208: 32-41.

Figura 4: Estados de perfusión cerebral definidos en relación al flujo sanguíneo cerebral (FSC). La perfusión cerebral normal corresponde a valor de FSC entre 55 y $100 \mathrm{ml} / 100 \mathrm{gr} / \mathrm{min}$. Cualquier valor menor a 55 es considerado un estado de hipoperfusión cerebral. Cuando la hipoperfusión no determina síntomas por disfunción neurológica, recibe el nombre de oligoemia (habitualmente con FSC entre 22 y 55 ml/100gr/min); cuando en el estado de hipoperfusión existen síntomas neurológicos, se denomina isquemia (habitualmente con FSC $<22 \mathrm{ml} / 100 \mathrm{gr} / \mathrm{min}$ ). Dentro de la isquemia se distingue la penumbra (tejido potencialmente recuperable, habitualmente con FSC entre 10 y $22 \mathrm{ml} / 100 / \mathrm{gr} / \mathrm{min}$ ) del core de infarto (tejido con daño irreversible, habitualmente con FSC $<10 \mathrm{ml} / 100 \mathrm{gr} / \mathrm{min}$ ).

Tabla 2. Cinco estados de perfusión cerebral, definidos por valores de FSC, VSC y TTM.

\begin{tabular}{|lllll|}
\hline ESTADO & $\begin{array}{l}\text { FSC } \\
(\mathrm{ml} / \mathrm{min} / 100 \mathrm{grs})\end{array}$ & $\begin{array}{l}\text { VSC } \\
(\mathrm{ml} / 100 \mathrm{grs})\end{array}$ & $\begin{array}{l}\text { TTM } \\
(\text { segundos })\end{array}$ & EJEMPLO \\
\hline Normal & $>55$ & $2-3$ & $<6 \mathrm{seg}$ & \\
Flujo lento & $\mathrm{N}$ & $\mathrm{N}$ & $\uparrow$ & Revascularización, colaterales \\
Hipoperfusión & $\downarrow(22-55)$ & $\mathrm{N}$ & $\uparrow$ & Estenosis proximal \\
Isquemia & $\downarrow \downarrow(<22)$ & $\mathrm{N} \circ \uparrow$ & $\uparrow(1,5$ veces $)$ & Penumbra \\
Infarto & $\downarrow \downarrow \downarrow(<10)$ & $\downarrow \downarrow(<2)$ & $\uparrow$ & Core de infarto \\
Hiperperfusión & $\mathrm{N} \circ \uparrow$ & $\uparrow(1,5-2$ veces $)$ & $\downarrow$ & Tumores de alto grado \\
& $\uparrow \uparrow$ & $\uparrow$ & Variable & Endarterectomía, estado postictal \\
\hline
\end{tabular}




\section{Indicaciones}

La principal indicación de la PTC es el estudio del infarto cerebral agudo con el objetivo de determinar penumbra isquémica susceptible de terapia de reperfusión, lo que hoy en día permite incluso extender la ventana terapéutica ${ }^{15,16,17}$. Sin embargo, existe abundante evidencia de su utilidad en otras situaciones clínicas entre las que destacamos los estados de hipo-hiperperfusión, determinación de grado tumoral en gliomas, vasculopatías y traumatismo encefálico ${ }^{1,3}$.

\section{Infarto cerebral}

Es el resultado de una estenosis u oclusión arterial, transitoria o permanente, con consecuente disminución del FSC hasta alterar la función neuronal ${ }^{18}$. Dado que es frecuente la presencia de un flujo residual procedente de los territorios de circulación colateral, es posible distinguir entre una zona central (núcleo o core del infarto) correspondiente a tejido cerebral infartado, no recuperable; versus una zona periférica (penumbra del infarto) correspondiente a tejido cerebral isquémico disfuncional, que es potencialmente recuperable mediante recanalización temprana ${ }^{19,20}$.

Los hallazgos de los mapas de perfusión encontrados en los casos de isquemia cerebral se explican por la falla en los mecanismos de autorregulación cerebral, con consecuente disminución de la presión de perfusión lo que conduce a una prolongación del TTM tanto en el core como en la zona de penumbra. Esto gatilla vasodiltación de los capilares de la zona de la lesión para mantener constante el FSC, así el VSC se mantiene constante o incluso aumentado, por lo que el tejido afectado puede mantener su viabilidad (penumbra isquémica). Cuando la autorregulación falla o no es capaz de compensar el descenso del FSC, el VSC disminuye y se genera un daño tisular irreversible (core o núcleo). En los mapas de colores la penumbra se identifica como una zona de discordancia (mismatch) entre el FSC y el VSC: FSC disminuido y VSC normal o incluso elevado ${ }^{2,3,4}$. En el caso del core el VSC se encuentra marcadamente disminuido, lo que permite hacer la diferencia con la penumbra. En las primeras horas del inicio de la isquemia es el TTM el parámetro que se altera de manera más precoz, sin embargo, no es capaz de distinguir core versus penumbra dado que se eleva en ambos ${ }^{4,7}$.

En el escenario de oclusión de un vaso principal seguida de recanalización, el VSC y el FSC aumentan en el territorio distal a la oclusión en comparación con el cerebro contralateral, fenómeno condicionado por vasodilatación del lecho vascular, correspondiente a un estado de hiperperfusión. Al interpretar la PTC en el contexto de un accidente cerebro-vascular agudo, se debe tener en cuenta este fenómeno, al igual que en escenarios de endarterectomía carotídea ${ }^{20}$.

Una situación particular es la diásquisis cerebelosa cruzada, que corresponde a una depresión en el metabolismo y la actividad neuronal del hemisferio cerebeloso contralateral a una lesión supratentorial, probablemente secundaria a la interrupción de la vía cortico-ponto-cerebelosa, que se manifiesta por hipoperfusión y que puede estar presente en aproximadamente un $20 \%$ de las PTC realizadas durante un accidente cerebro-vascular agudo. En las etapas crónicas, puede haber pérdida de volumen asociada en el hemisferio cerebeloso comprometido, conocida como atrofia cerebelosa cruzada ${ }^{21,22}$.

En la figura 5 presentamos un paciente con infartos múltiples del hemisferio cerebral izquierdo (secundarios a oclusión carotidea) con diásquisis cerebelosa cruzada del hemisferio cerebral derecho.

\section{Vasculopatías}

La presencia de estenosis arteriales son hallazgos comunes entre las vasculopatías inflamatorias (ya sea vasculitis primaria o secundarias), vasculopatías no inflamatorias y en el vasoespasmo. Si bien las alteraciones de perfusión corresponden a hallazgos indirectos, su conocimiento puede ser de utilidad para el proceso diagnóstico, ya que proporciona información relevante acerca del estado hemodinámico cerebral, especialmente en la identificación de tejido infartado y de tejido isquémico potencialmente en riesgo, incluso sin estenosis arterial demostrada con estudios no invasivos ${ }^{23}$. Para el caso del vasoespasmo se ha demostrado su utilidad en la anticipación de la isquemia cerebral tardía (ICT) en contexto de hemorragia subaracnoídea aneurismática, donde los pacientes que presentaban alteraciones de la PTC registraron un riesgo 23 veces mayor de ICT 25 .

En la figura 6 presentamos un paciente con una vasculitis infecciosa e hipoperfusión cerebral global secundaria.

\section{Control de cirugía de revascularización cerebral}

La cirugía de revascularización cerebral -ya sea mediante revascularización directa con bypass extraintracraneal o revascularización indirecta mediante sinangiosis- corresponde a un grupo de técnicas microquirúrgicas útiles para el tratamiento de la enfermedad isquémica intracraneal esteno-oclusiva (ej.: enfermedad de Moyamoya), aneurismas intracraneales complejos que requieren oclusión de la arteria proximal y algunos tumores invasivos de la base del cráneo. Los estudios de PTC son útiles para la evaluación del resultado terapéutico y para el seguimiento de la evolución. Sin embargo, debe considerarse en estos pacientes algunas limitaciones derivadas de la selección del input arterial y la redistribución del flujo producto de la(s) anastomosis (retraso y dispersión del bolo); estas limitaciones pueden ser superadas mediante un análisis detallado y contextualizado que considere los estudios vasculares previos y contemporáneos del paciente ${ }^{24}$. 


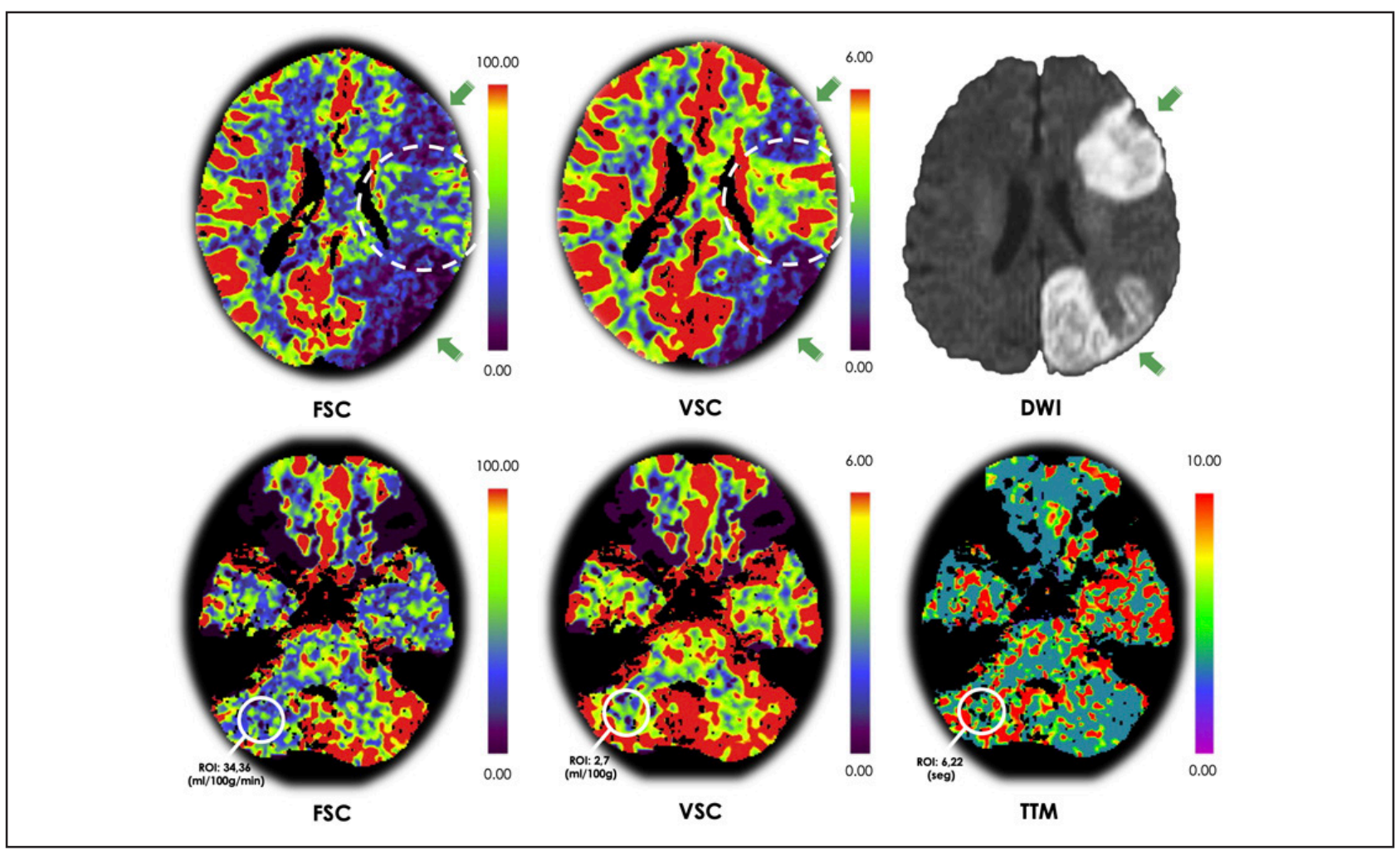

Figura 5: Hombre de 62 años con oclusión de la arteria carótida interna izquierda. Infartos frontal y parieto-occipital izquierdos, con disminución de los valores de FSC y VSC, en concordancia con las zonas de restricción en la RM secuencia difusión (flechas verdes). Nótese la región opercular izquierda (entre ambos infartos), con disminución del valor de FSC y preservación del valor de VSC, compatible con penumbra isquémica (círculos blancos con línea incompleta). Se observa además hipoperfusión del hemisferio cerebeloso derecho (FSC con valor disminuido, VSC con valor normal y TTM prolongado) (círculos pequeños blancos con línea continua). Esta alteración es consistente con diásquisis cerebelosa cruzada.

En la figura 7 presentamos a un paciente con enfermedad de Moyamoya, en control postoperatorio de revascularización cerebral mediante anastomosis de arteria temporal al segmento M3 de la arteria cerebral media, además de sinangiosis arteriodural.

\section{Tumores}

Los estudios de perfusión cerebral son útiles para diferenciar lesiones pseudotumorales de tumores verdaderos, donde las primeras habitualmente cursan, con VSC bajo y los segundos con VSC alto, sin embargo, la principal utilidad de la PTC consiste en la aproximación al grado tumoral en las neoplasias gliales, dado que la neovascularización de los tumores de alto grado condiciona un incremento del VSC y a su vez aumenta la permeabilidad de la barrera hemato-encefálica (esta última evaluada mediante el PEF) $)^{1,26}$. Este hecho explica la regla de que los tumores de mayor grado tienden a tener valores más altos de VSC, aunque son conocidas las excepciones del oligodendroglioma y el astrocitoma pilocítico, dadas las características histológicas particulares de su vasculatura ${ }^{27}$.

Adicionalmente se conoce que el linfoma del SNC en algunos casos puede presentar hiperperfusión similar a los gliomas de alto grado, situación a considerar en el diagnóstico diferencial. La PTC también es una herramienta útil para evaluar los tumores tratados y distinguir entre radionecrosis versus recurrencia tumoral, la primera hipoperfundida y la segunda con áreas de hiperperfusión patológicas ${ }^{1,27}$.

En la figura 8 presentamos un astrocitoma de bajo grado, con valores de VSC y PEF no elevados. En contraste, en la figura 9 presentamos un glioblastoma, con valores de VSC y PEF elevados.

\section{Cambios hemodinámicos postconvulsivos}

En el contexto de convulsiones o estatus convulsivos, los mapas de PTC pueden mostrar hiperperfusión en las regiones corticales afectadas, lo que permite diferenciar un accidente cerebro-vascular de una parálisis postconvulsiva (parálisis de Todd) ${ }^{28,29}$.

En la figura 10 presentamos un paciente con estatus convulsivo e hiperperfusión cortical secundaria. 


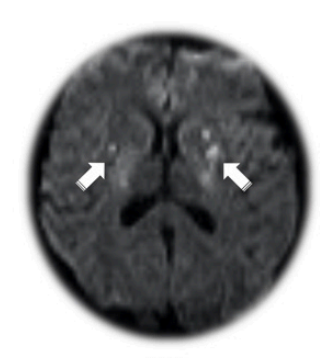

DWI

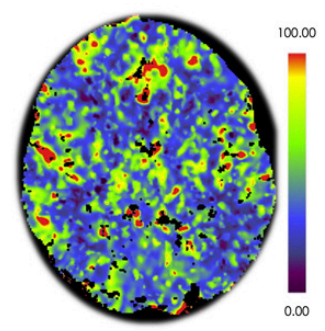

FSC

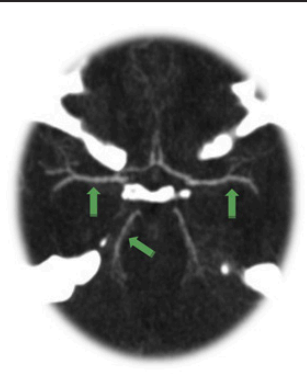

Angio-TC

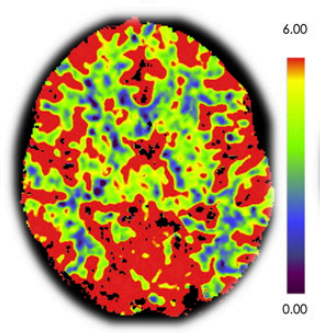

vsC

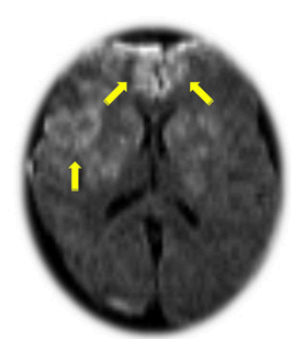

DWI control

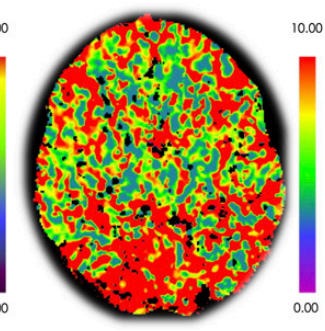

$\pi \mathrm{M}$

Figura 6: Mujer de 32 años, VIH (+), cursando meningitis criptocócica. La primera RM secuencia difusión muestra focos de restricción ganglionares basales bilaterales (pseudoquistes gelatinosos) (flechas blancas). Una Angio-TC muestra múltiples zonas de estenosis, con aspecto "arrosariado" de los segmentos proximales de las distintas arterias cerebrales (flechas verdes), compatible con una vasculitis secundaria al proceso inflamatorio-infeccioso meníngeo. Una segunda RM secuencia difusión, realizada 5 días después de la primera RM, muestra múltiples zonas de isquemia córtico-subcorticales en territorio de la circulación anterior (de predominio a derecha). En el estudio de perfusión por TC se observa disminución generalizada del FSC, valores normales de VSC y marcada prolongación generalizada del TTM, consistente con una hipoperfusión global (multifocal). Nótese la tendencia a los tonos azules en el mapa de FSC (especialmente en la corteza), correspondiente a bajo flujo; y la tendencia a los tonos rojos en el mapa de TTM, correspondiente a prolongación del tiempo.

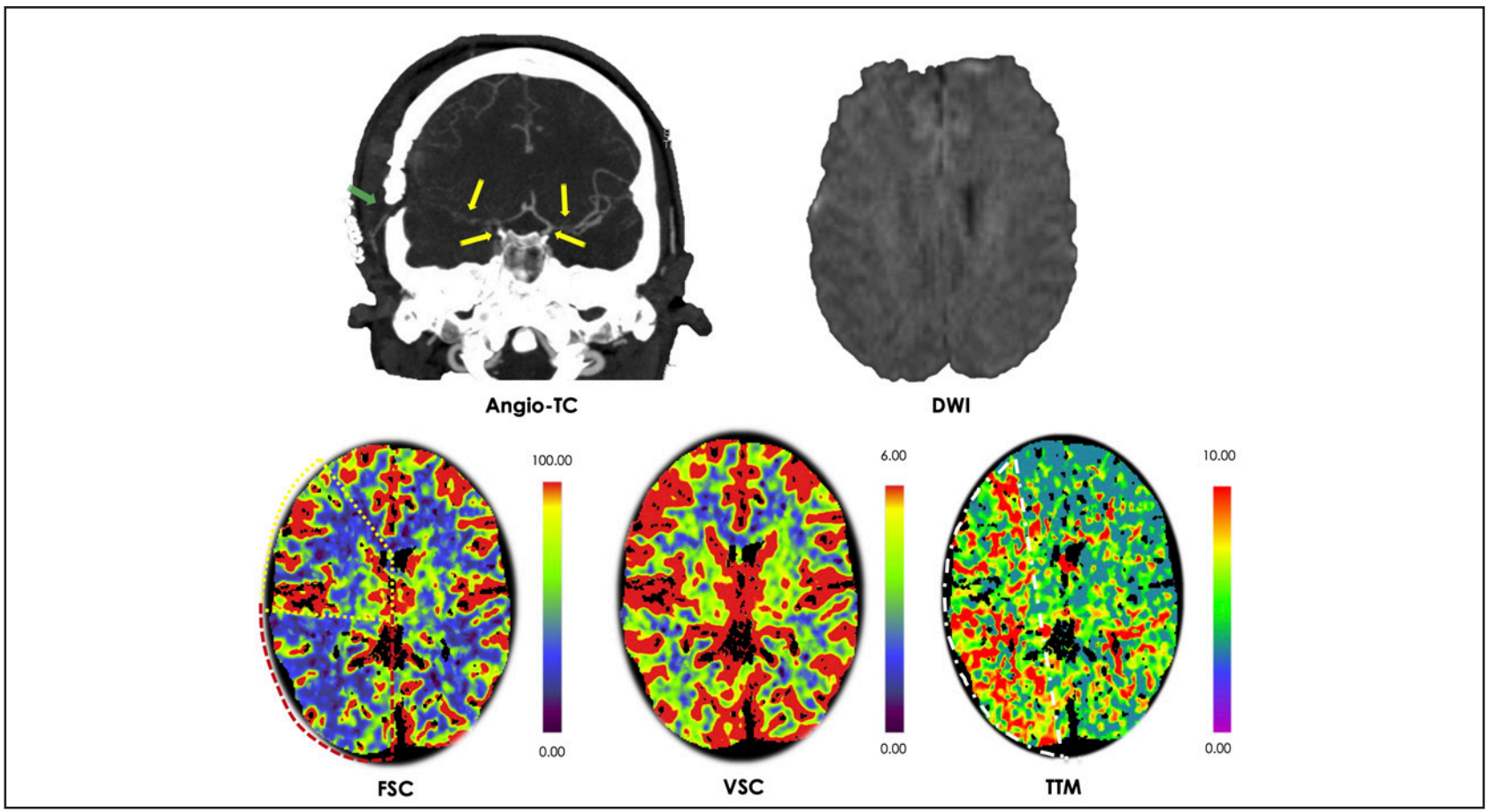

Figura 7: Mujer de 36 años con enfermedad de Moyamoya. La Angio-TC demuestra compromiso esteno-oclusivo de las arterias carótidas internas (mayor a derecha) y del segmento M1 de las arterias cerebrales medias (flechas amarillas). Se identifica también al lado derecho la anastomosis quirúrgica entre territorio vascular carotideo externo y territorio de la arteria cerebral media. Una RM secuencia difusión postoperatoria no muestra lesiones isquémicas. El estudio de perfusión por TC muestra prolongación del TTM al lado derecho, en territorio de ACM y ACP (línea blanca discontinua); el FSC presenta valor disminuido a nivel parieto-occipital, indicativo de hipoperfusión (línea roja discontinua), y preservación de su valor a nivel frontal, indicativo de flujo lento (línea amarilla discontinua). El valor de VSC se encuentra globalmente preservado. 


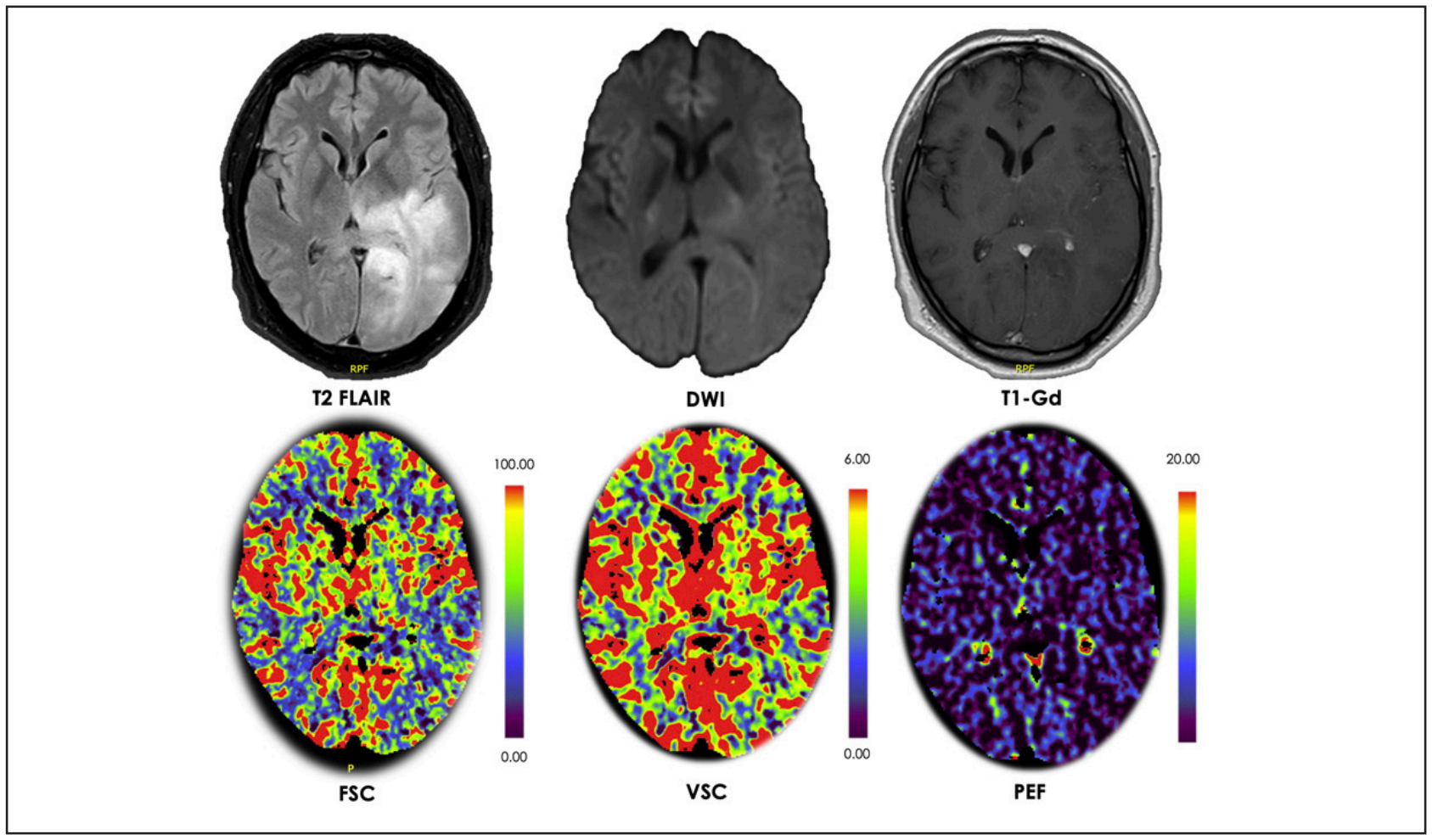

Figura 8: Hombre de 42 años con alteración del lenguaje. La RM muestra una masa intraaxial témporo-parietal izquierda, con efecto de masa, sin restricción a la difusión ni realce con el Gadolinio. El estudio de PTC no demuestra aumento de los parámetros FSC, VSC ni PEF en relación a esta lesión. El diagnóstico histológico de la lesión fue un astrocitoma de bajo grado (OMS grado II).

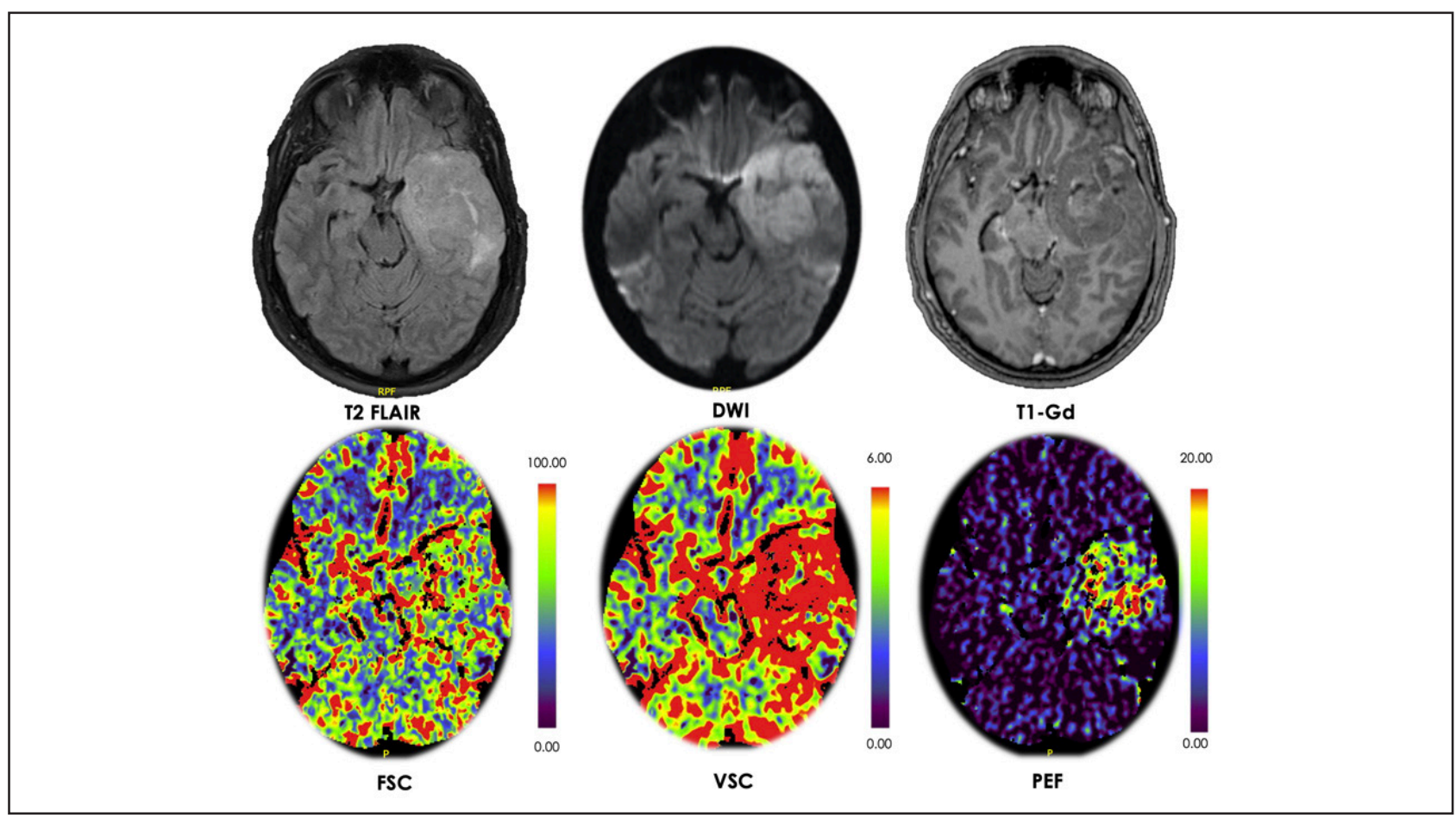

Figura 9: Hombre de 34 años con alteración de memoria. La RM muestra una masa intraaxial temporal izquierda, con efecto de masa, zonas de restricción a la difusión y leve realce heterogéneo con el Gadolinio. El estudio de PTC demuestra aumento del valor de VSC y PEF en relación a esta lesión (traduciendo neovascularización y disrupción de la barrera hematoencefálica respectivamente); el valor de FSC se mantiene sin elevación significativa. El diagnóstico histológico de la lesión fue un glioblastoma (OMS grado IV). 

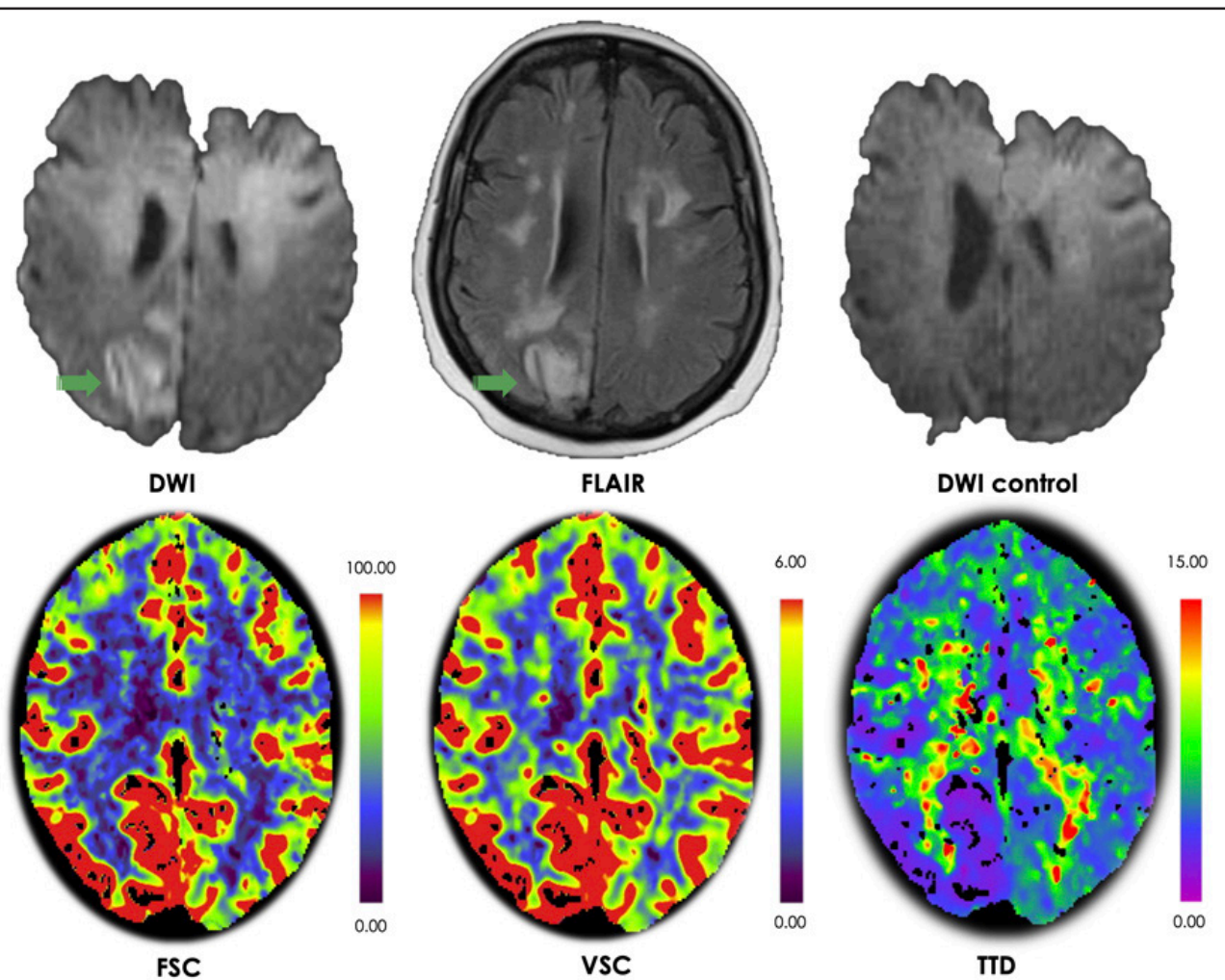

Figura 10: Mujer de 58 años con estatus convulsivo. La RM secuencia difusión muestra una zona de restricción cortical parietal mesial derecha, con hiperseñal T2 FLAIR (flechas verdes). En el estudio de PTC esta zona demuestra elevación de los valores de FSC y VSC, con acortamiento del TTD, correspondiente a hiperperfusión postictal. Una segunda RM secuencia difusión, realizada 7 días después, muestra resolución de la restricción, sin evidencia de una secuela.

\section{Traumatismo encefálico}

En el contexto de un trauma la PTC tiene una contribución pronostica significativa, dado que aporta elementos a la TC cerebral sin contraste y se correlaciona de manera proporcional con la puntuación de Glasgow ${ }^{1,30}$. La perfusión cerebral normal o la hiperemia (valores altos de VSC y FSC) se asocian a un resultado favorable, mientras que la hipoperfusión presente en el TEC severo (valores bajos de VSC y FSC) se correlacionan con un resultado desfavorable. Además de la gravedad inicial de la injuria, la hipertensión intracraneal secundaria es el principal factor que determina el resultado final en pacientes con lesiones traumáticas encefálicas y suele ser mortal con valores sostenidamente elevados ${ }^{30,31}$. En este escenario, la PTC evidencia valores VSC y FSC absolutos bajos con valores de TTM significativamente altos, correspondientes a una hipoperfusión extensa, posiblemente debida a isquemia cerebral consecutiva a la hipertensión intracraneal. El edema cerebral también puede evidenciar alteraciones en los estudios de perfusión: En el edema citotóxico la perfusión cerebral disminuye y en el edema vasogénico puede aumentar o disminuir. La PTC tiene una mayor sensibilidad para el diagnóstico de las contusiones cerebrales en comparación con la TC cerebral sin contraste, ya que permite precozmente delimitar la extensión completa del área lesionada (antes de la aparición de hemorragia y/o edema) ${ }^{1,30}$. Finalmente, la PTC puede evidenciar la alteración de la perfusión cerebral en las proximidades de las colecciones yuxtadurales. Esta alteración tiende a ser más pronunciada en los hematomas epidurales que en los subdurales ${ }^{30,31}$.

En la figura 11 mostramos un paciente con TEC complicado (contusión parenquimatosa cerebral y hemorragia subaracnoidea traumática), que presenta un área de hipoperfusión temporal anterior izquierda, correspondiente al área de contusión parenquimatosa. 


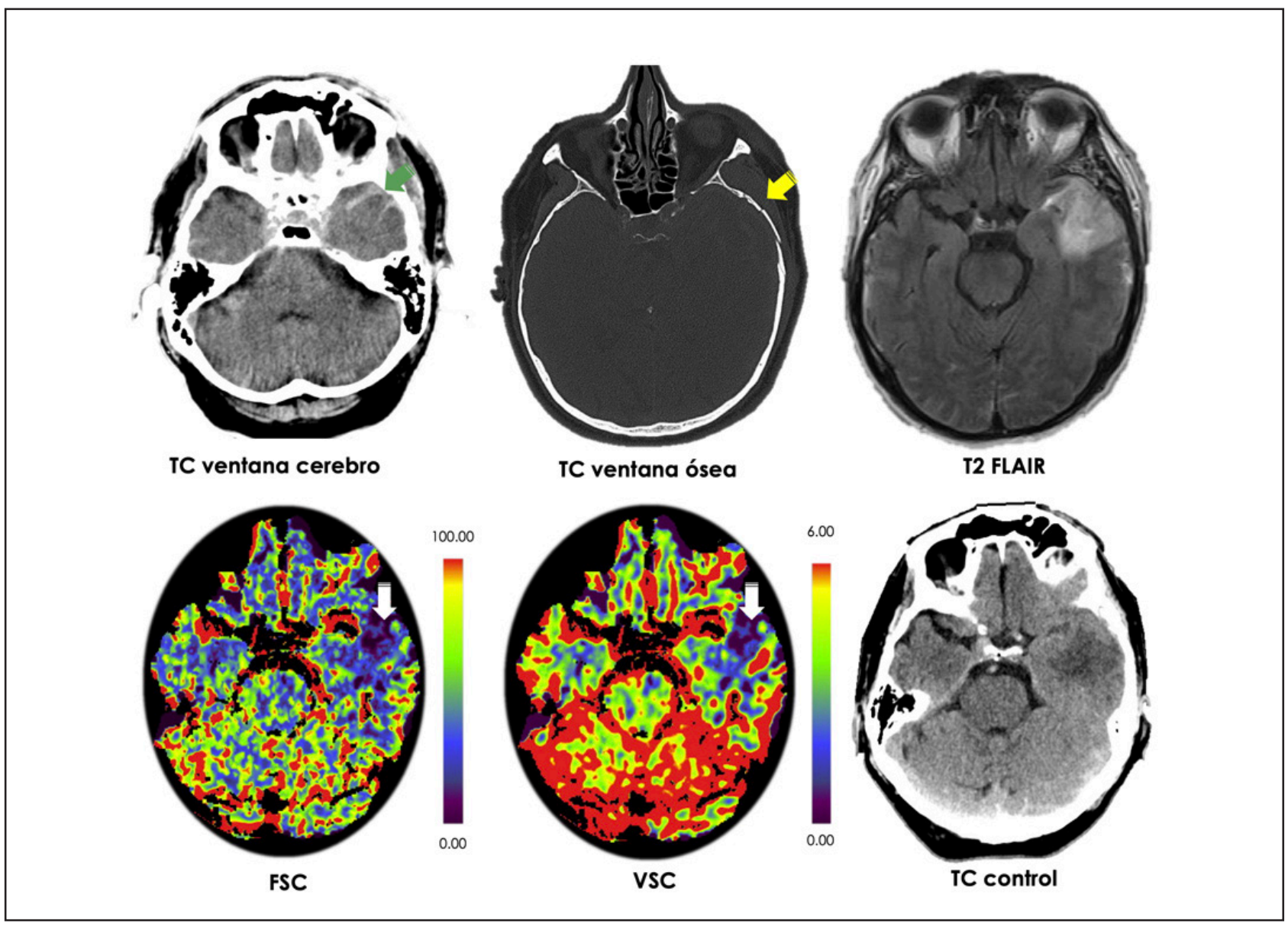

Figura 11: Hombre de 81 años, trauma cráneo-encefálico por accidente automovilístico. La TC de cerebro realizada al ingreso muestra HSA temporal izquierda (flecha verde), con fractura de la escama temporal adyacente (flecha amarilla). La PTC simultánea muestra hipoperfusión en la región temporal anterior izquierda (bajo valor de FSC y VSC, flechas blancas). Una RM secuencia T2 FLAIR confirma la presencia de un foco de contusión parenquimatosa temporal anterior izquierdo. La TC de cerebro de control, realizada 72 horas después del ingreso muestra la consolidación del edema del foco de contusión parenquimatosa.

\section{Conclusión}

La PTC es una técnica útil y ampliamente disponible para evaluar la perfusión cerebral. La principal aplicación de la PTC es la evaluación de pacientes con infarto cerebral agudo, sin embargo, existen indicaciones adicionales, cuya utilidad fue ejemplificada en este artículo, tales como estados de hipoperfusión (ej: vasculopatías esteno-oclusivas), estados de hiperperfusión (ej: estado posreperfusión cerebral, estado posconvulsivo), control posoperatotio de cirugía de revascularización cerebral, determinación del grado tumoral en gliomas y evaluación de traumatismo encefálico. En todas estas condiciones el estudio de perfusión puede ser útil, proporcionando información relevante para el diagnóstico y tratamiento.

El análisis de los mapas de perfusión cerebral re- quiere el conocimiento de los parámetros fisiológicos, los antecedentes clínicos y los estudios complementarios, además implica una valoración integrada de los distintos resultados cuantitativos y cualitativos disponibles. La aproximación sistematizada es importante para hacer un diagnóstico correcto. Proponemos una tabla con puntos de corte que definen cinco estados de perfusión cerebral, lo cual facilita la aproximación sistemática a estos estudios.

Creemos que la PTC cerebral es una técnica que se puede implementar en la mayoría de los hospitales del país que cuenten con equipo de TC. Si en los hospitales se cuenta con personal capacitado, es posible un rápido posprocesamiento, obteniendo mapas de perfusión en pocos minutos luego de la finalización de la adquisición de datos. 


\section{Referencias}

1. Wintermark M, Sincic R, Sridhar D, Chien JD. Cerebral perfusion CT: Technique and clinical applications. J Neuroradiol. 2008; 35: 253-260.

2. Benitez-Vazquez A, Martínez M, Sanchez R, Murias E. Aspectos prácticos de la TC de Perfusión cerebral. SERAM. 2012; 450: 1-66.

3. Hoeffner E, Case I, Jain R, Gujar SK, Shah G, Deveikis JP, et al. Cerebral Perfusion CT: Technique and Clinical Applications. Radiology. 2004; 231: 632-644.

4. Leiva-Salinas C, Provenzale JM, Wintermark M. Responses to the 10 most frequently asked questions about perfusion CT. AJR Am J Roentgenol. 2011; 196: 53-60.

5. Miles KA, Griffiths MR. Perfusion CT: a worthwhile enhancement? The British Journal of Radiology. 2003; 76: 220-231.

6. Calamante F, Christensen S, Desmond P, et al. The Physiological Significance of the Time-to-Maximum (Tmax) Parameter in Perfusion MRI. Stroke. 2010; 41: 1169-1174.

7. Lucas E, Sánchez E, Gutiérrez A, Mandly A, Ruiz E, Flórez AF, et al. CT protocol for acute stroke: tips and tricks for general radiologists. Radiographics. 2008; 6: 1673-1687.

8. Othman A, Afat S, Brockmann MA, Nikoubashman O, Brockmann C, Nikolaou K, et al. Radiation dose reduction in perfusion $C T$ imaging of the brain: $A$ review of the literature. J Neuroradiol. 2015; 43: 1-5.

9. Smith AB, Dillon WP, Gould R, Wintermark M. Radiation dose-reduction strategies for neuroradiology CT protocols. AJNR Am J Neuroradiol. 2007; 9: 1628-1632.

10. Mori S, Obata T, Nakajima N, Ichihara N, Endo M. Volumetric perfusion CT using prototype 256-detector row CT scanner: preliminary study with healthy porcine model. AJNR Am J Neuroradiol. 2005; 26: 2536-2541.

11. Tomandl B, Klotz E, Handschu R, Stemper B, Reinhardt F, Huk WJ, et al. Comprehensive imaging of ischemic stroke with multisection CT. Radiographics. 2003; 3: 565-592.

12. Sanelli P, Lev M, Eastwood J, Gonzalez RG, Lee TY. The effect of varying user-selected input parameters on quantitative values in CT perfusion maps. Acad Radiol. 2004; 11: 1085-1092.

13. Mangla R, Ekhom S, Jahromi B.S, et al. CT perfusion in acute stroke: Know the mimics, potential pitfalls, artifacts, and technical errors. Emerg Radiol 2014; 21: 49-65.

14. Boned S, Padroni M, Rubiera M, et al. Admission CT perfusion may overestimate initial infarct core: the ghost infarct core concept. Journal of Neurolnterventional Surgery 2017; 9: 66-69.

15. Lui YW, Tang ER, Allmendinger AM, Spektor V. Evaluation of CT perfusion in the setting of cerebral ischemia: patterns and pitfalls. AJNR Am J Neuroradiol. 2010; 9: 1552-1563.

16. Lev MH, Segal AZ, Farkas J, Hossain S, Putman C, Hunter GJ, et al. Utility of perfusion-weighted CT imaging in acute middle cerebral artery stroke treated with intra-arterial thrombolysis: prediction of infarct volume and clinical outcome. Stroke. 2001; 32: 20212028.
17. Wintermark $M$, Reichhart $M$, Thiran JP, Reichhart M, Thiran JP, Maeder P, et al. Prognostic accuracy of cerebral blood flow measurement by perfusion computed tomography, at the time of emergency room admission, in acute stroke patients. Ann Neurol. 2002; 51: 417-432.

18. Koenig M, Klotz E, Luka B, Venderink DJ, Spittler JF, Heuser L. Perfusion CT of the brain: diagnostic approach for early detection of ischemic stroke. Radiology. 1998; 209: 85-93.

19. Von Kummer R, Allen KL, Holley R, Bozzao L, Bastianello $S$, et al. Acute stroke: usefulness of early CT findings before thrombolytic therapy. Radiology. 1997; 205: 327-333.

20. Srinivasan A, Goyal M, Al Azri F, Lum C. State of the art imaging of acute stroke. Radiographics. 2006; 26: 75-95.

21. Han S, Wang X, Xu K, Hu C. Crossed Cerebellar Diaschisis: Three Case Reports Imaging Using a Tri-Modality PET/CT-MR System. Medicine. 2016; 2: 1-4.

22. Tien R, Ashdown B. Crossed cerebellar diaschisis and crossed cerebellar atrophy: correlation of MR findings, clinical symptoms, and supratentorial diseases in 26 patients. AJR Am J Roentgenol. 1992; 158: 1155-1159.

23. Yuh W, Ueda T, Maley J. Perfusion and Diffusion Imaging: A Potential Tool for Improved Diagnosis of CNS Vasculitis. AJNR Am J Neuroradiol. 1999; 20: 87-89.

24. Zhang J, Wang J, Geng D, Li Y, Song D, Gu Y. Whole-brain CT perfusion and CT angiography assessment of Moyamoya disease before and after surgical revascularization: preliminary study with 256-slice CT. PLoS One. 2013; 8: 1-6.

25. Mir D, Gupta A, Dunning A, Puchi L, Robinson C, Epstein HA, et al. CT perfusion for detection of delayed cerebral ischemia in aneurysmal subarachnoid hemorrhage: A systematic review and meta-analysis. American Journal of Neuroradiology. 2014; 35: 866871.

26. Cenic A, Nabavi D, Gellb A. A CT method to measure hemodynamics in brain tumors: Validation and application of cerebral blood flow maps. AJNR Am J Neuroradiol. 2000; 21: 462-470.

27. Cianfoni A, Cha S,Bradley W. Quantitative measurement of blood brain barrier permeability using perfusion-CT in extra axial brain tumors. J Neurorradiol. 2006; 33: 164-168.

28. Royter V, Paletz L, Waters MF. Stroke vs. status epilepticus: A case report utilizing CT perfusion. J Neurol Sci. 2008; 266: 174-176.

29. Masterson K, Vargas MI, Delavelle J. Postictal deficit mimicking stroke: role of perfusion CT. J Neuroradiol. 2009; 36: 48-51.

30. Wintermark M, Guy van Melle M, Schnyder P. Admission Perfusion CT: Prognostic Value in Patients with Severe Head Trauma. Radiology. 2004; 1: 211-220.

31. Lannoo E, Van Rietvelde F, Colardyn F, Lemmerling M, Vandekerckhove T, Jannes $C$, et al. Early predictors of mortality and morbidity after severe closed head injury. J Neurotrauma. 2000; 17: 403-414. 